\title{
APPROXIMATIONS FOR LARGE DEFLECTION OF A CANTILEVER BEAM*
}

BY K. E. BISSHOPP (Rensselaer Polytechnic Institute)

\begin{abstract}
Rational approximations for the end slope and deflection of a cantilever beam are derived from the exact solution in terms of elliptic integrals [1] by asymptotic methods.

1. Theory. As an example of the consistent evaluation of small quantities, the original objective of this note was to obtain the elementary formulas for the cantilever beam by linearizing the elliptic integral solution. By combining the first and second order approximations for small quantities with obvious properties of the deflected configuration it is possible to construct an approximate theory which is valid for moderately large deflections.

For the case of a simple cantilever beam of length $l$ with a single vertical end load $P$, the nonlinear solution is known to be expressible in terms of elliptic integrals with parameter $\theta_{0}$ which is the end slope of the beam. The pertinent relations are:

$$
\begin{gathered}
B=\frac{P l^{2}}{E I} ; \quad \sqrt{ } B=\int_{u_{0}}^{\pi / 2} \frac{d u}{\left(1-k^{2} \sin ^{2} u\right)^{1 / 2}}=K\left(k, \frac{\pi}{2}\right)-K\left(k, u_{0}\right) ; \\
\frac{y_{0}}{l}=1-\frac{2}{\sqrt{ } B} \int_{u_{0}}^{\pi / 2}\left(1-k^{2} \sin ^{2} u d u\right)^{1 / 2}=1-\frac{2}{\sqrt{ } B}\left[E\left(k, \frac{\pi}{2}\right)-E\left(k, u_{0}\right)\right] ; \\
\frac{x_{0}}{l}=\left(\frac{2 \sin \theta_{0}}{B}\right)^{1 / 2} ; \quad k^{2}=\frac{1+\sin \theta_{0}}{2} ; \quad \sin u_{0}=\frac{1}{\left(1+\sin \theta_{0}\right)^{1 / 2}}, 0 \leq \theta_{0} \leq \frac{\pi}{2},
\end{gathered}
$$
\end{abstract}

where $x_{0}, y_{0}$ are the coordinates of the loaded end of beam and $K, E$ are elliptic integrals of first and second kind, respectively.

The inequality $\theta_{0} \ll 1$ for the linear case shows that $u_{0} \simeq(\pi / 2)$ so that an approximation for $w=(\pi / 2)-u_{0}$ can be derived from Eq. (3):

$$
\sin w=\left(\frac{\sin \theta_{0}}{1+\sin \theta_{0}}\right)^{1 / 2} \simeq\left(\sin \theta_{0}\right)^{1 / 2}\left(1-\frac{1}{2} \theta_{0}+\frac{3}{8} \theta_{0}^{2}\right)
$$

since

$\frac{1}{\left(1 \pm \sin \theta_{0}\right)^{1 / 2}}=\sec \theta_{0}\left(\cos \frac{\theta_{0}}{2} \mp \sin \frac{\theta_{0}}{2}\right)$

$$
=1 \mp \frac{1}{2} \theta_{0}+\frac{3}{8} \theta_{0}^{2} \mp \frac{11}{48} \theta_{0}^{3} \pm \cdots 0 \leq \theta_{0} \leq 1 .
$$

As the angle $w \ll 1$, it would ordinarily be assumed that $w$ could be approximated with sufficient accuracy by $\left(\sin \theta_{0}\right)^{1 / 2}\left(1-\frac{1}{2} \theta_{0}+\frac{3}{8} \theta_{0}^{2}\right)$; however, this expression is not even correct to first-order small quantities. Now an arc is always longer than its chord,

* Received October 7, 1971. 
which suggests that the arc sin expansion be applied to Eq. (4). Three terms give the second-order approximation

$$
w=\left(\sin \theta_{0}\right)^{1 / 2}\left(1-\frac{1}{3} \theta_{0}+\frac{1}{5} \theta_{0}^{2}\right) .
$$

If $\theta_{0}^{3}$ is neglected, we obtain

$$
u_{10}=\frac{\pi}{2}-\left(\sin \theta_{0}\right)^{1 / 2}\left(1-\frac{1}{3} \theta_{0}+\frac{1}{5} \theta_{0}^{2}\right) .
$$

It is also necessary to construct second-order approximations for the integrands of Eqs. (1) and (2), as follows: $\left(1-k^{2} \sin ^{2} u\right)^{1 / 2}=\left(1-k^{2}+k^{2} \cos ^{2} u\right)^{1 / 2}$, where $u \simeq$ $(\pi / 2)$. A series quotient and Eq. (5) yield:

$$
k^{2} /\left(1-k^{2}\right)=\left(1+\sin \theta_{0}\right) /\left(1-\sin \theta_{0}\right) \simeq 1+2 \theta_{0}+2 \theta_{0}^{2}
$$

and

$$
\begin{aligned}
& \left(1-k^{2}\right)^{1 / 2} \simeq \frac{1}{2} \sqrt{ } 2\left(1-\frac{1}{2} \theta_{0}-\frac{1}{8} \theta_{0}^{2}\right), \\
& \frac{1}{\left(1-k^{2}\right)^{1 / 2}} \simeq \sqrt{ } 2\left(1+\frac{1}{2} \theta_{0}+\frac{3}{8} \theta_{0}^{2}\right) .
\end{aligned}
$$

Expansion of Eqs. (1) and (2) by the binomial theorem gives

$$
\begin{aligned}
\sqrt{ } B & =\sqrt{ } 2\left(1+\frac{1}{2} \theta_{0}+\frac{3}{8} \theta_{n}^{2}\right) \int_{u_{0}}^{\pi / 2}\left[1-\frac{1+2 \theta_{0}}{2} \cos ^{2} u+\frac{3}{8}\left(1+4 \theta_{0}\right) \cos ^{4} u\right] d u, \\
\frac{y_{0}}{l} & =1-\frac{\sqrt{ } 2\left(1-\frac{1}{2} \theta_{0}-\frac{1}{8} \theta_{0}^{2}\right)}{\sqrt{ } B} \int_{u_{0}}^{\pi / 2}\left[1+\frac{1+2 \theta_{0}}{2} \cos ^{2} u-\frac{1}{8}\left(1+4 \theta_{0}\right) \cos ^{4} u\right] d u .
\end{aligned}
$$

The transformation $u=(\pi / 2)-\theta$ applies here and at the same time shows that three terms of the binomial expansion are sufficient for a second-order approximation. Then Eq. (11) becomes

$$
\begin{aligned}
\sqrt{ } B=\sqrt{ } 2\left(1+\frac{1}{2} \theta_{0}+\frac{3}{8} \theta_{0}^{2}\right) \\
\cdot \int_{0}^{\left(\sin \theta_{0}\right)^{2 / 3\left(1-1 / 3 \theta_{0}+1 / 5 \theta_{0}\right)}}\left[1+\frac{1+2 \theta_{0}}{2} \sin ^{2} \theta+\frac{3}{8}\left(1+4 \theta_{0}\right) \sin ^{4} \theta\right] d \theta
\end{aligned}
$$

and a similar equation for $y_{0} / l$. For $\theta \ll 1$ this integral can be approximated by

$$
J_{1}=\int_{0}^{\infty}\left[1-\frac{1+2 \theta_{0}}{2}\left(\theta-\frac{1}{6} \theta^{3}\right)^{2}+\frac{3}{8}\left(1+4 \theta_{0}\right)\left(\theta-\frac{1}{6} \theta^{3}\right)^{4}\right] d \theta .
$$

After terms of order three and higher are neglected, we obtain

$$
J_{1} \simeq\left(\sin \theta_{0}\right)^{1 / 2}\left(1-\frac{1}{2} \theta_{0}+\frac{17}{120} \theta_{0}^{2}\right)
$$

and thus

$$
\begin{aligned}
\sqrt{ } B & =\left(2 \sin \theta_{0}\right)^{1 / 2}\left(1+\frac{1}{2} \theta_{0}+\frac{3}{8} \theta_{0}^{2}\right)\left(1-\frac{1}{2} \theta_{0}+\frac{17}{120} \theta_{0}^{2}\right) \\
& \simeq\left(2 \sin \theta_{0}\right)^{1 / 2}\left(1+\frac{4}{15} \theta_{0}^{2}\right) ; \quad \theta_{0}<B / 2 .
\end{aligned}
$$

1 The term $2 \theta_{0}^{2}$ can be neglected, since it yields only third-order terms. 
Likewise for $y_{0} / l$,

$$
J_{2} \simeq\left(\sin \theta_{0}\right)^{1 / 2}\left(1-\frac{1}{6} \theta_{0}+\frac{37}{120} \theta_{0}^{2}\right) \simeq\left(\sin \theta_{0}\right)^{1 / 2}\left(1-\frac{1}{6} \theta_{0}+\frac{1}{3} \theta_{0}^{2}\right)
$$

and thus

$$
\frac{y_{0}}{l}=1-\frac{\left(2 \sin \theta_{0}\right)^{1 / 2}\left(1-\frac{1}{2} \theta_{0}-\frac{1}{8} \theta_{0}^{2}\right)\left(1-\frac{1}{6} \theta_{0}+\frac{37}{120} \theta_{0}^{2}\right)}{\left(2 \sin \theta_{0}\right)^{1 / 2}\left(1+\frac{4}{15} \theta_{0}^{2}\right)} \simeq \frac{2}{3} \theta_{0}+0\left(\theta_{0}^{3}\right)<\frac{B}{3} .
$$

Eqs. (15) and (17) reduce to the linear theory when $\theta_{0}^{3}$ is neglected, which explains the remarkable accuracy of the classical theory for $B<1$.

2. Second-order modified theory. Reference [1] shows that as $\theta_{0} \rightarrow \pi / 2, B \rightarrow \infty$. Therefore it seems reasonable to assume that

$$
B \simeq 2 \sin \theta_{0} /\left(1-\left(\frac{2 \theta_{0}}{\pi}\right)^{2}\right)
$$

is an acceptable approximation for $B$ when $\theta_{0}<\pi / 2$. That this is the case can be justified by substituting Eq. (18) into Eq. (3) and comparing the computed values of

$$
x_{0} / l \simeq\left(1-\left(\frac{2 \theta_{0}}{\pi}\right)^{2}\right)^{1 / 2}
$$

with the exact theory. The independent variable of Eqs. (18) and (19) is $\theta_{0}$ and when it is eliminated numerically, the resulting values of $B$ and $x_{i} / l$ agree well with the exact theory (see Table I). For purposes of computation, the independent variable is not $\theta_{0}$ but $B$ which can only be obtained by inverting the transcendental equation (18). However, an asymptotic approximation for $\theta_{0}$ in terms of $B$ can be constructed by assuming first that $\theta_{0} \ll 1$, so that $\sin \theta_{0} \simeq \theta_{0}-\theta_{0}^{3} / 6$. When this relation and the first-order approximation $2 \theta_{0}=B+\epsilon$ are substituted into Eq. (15) it can be solved for values of $B<1$ with sufficient accuracy that

$$
\theta_{0} \simeq \frac{1}{2} B\left(1-\frac{1}{12} B^{2}\right)
$$

which shows that the $\left(B, \theta_{0}\right)$ curve has zero curvature at the origin. For small $B$, the factor

$$
1-\frac{1}{12} B^{2} \simeq \frac{12}{B^{2}+12}
$$

\section{TABLE I}

\begin{tabular}{ccccc}
\hline$\theta_{0}$ & $\mathrm{~B}$ & $x_{0} / l$ & $x_{0} / l([1])$ \\
\hline 0 & 0. & 1.00 & 1.00 \\
$15^{\circ}$ & 0.53 & 0.99 & 0.98 \\
$30^{\circ}$ & 1.12 & 0.94 & 0.93 \\
$45^{\circ}$ & 1.90 & 0.87 & 0.85 \\
$60^{\circ}$ & 3.13 & 0.75 & 0.73 \\
$75^{\circ}$ & 6.34 & 0.55 & 0.54 \\
$90^{\circ}$ & $\infty$ & 0.00 & 0.00 \\
& & (Slide-rule computations) & \\
\hline
\end{tabular}


TABLE II

\begin{tabular}{ccccc}
\hline $\mathrm{B}$ & $\theta_{0}^{0}[$ Eq. $(18)]$ & $\theta_{0}^{0}[$ Eq. $(20)]$ & $x_{0} / l$ & $x_{0} / l([1])$ \\
\hline 0.0 & 0 & 0 & 1.00 & 1.00 \\
0.5 & 14.1 & 14.1 & 0.99 & 0.98 \\
1.0 & 27.0 & 27.0 & 0.97 & 0.95 \\
2.0 & 46.5 & 46.2 & 0.86 & 0.84 \\
3.0 & 58.5 & 57.0 & 0.76 & 0.74 \\
6.0 & 74.0 & 74.2 & 0.56 & 0.56 \\
9.0 & 79.7 & 82.0 & 0.47 & 0.47 \\
$\infty$ & 90.0 & 90.0 & 0.00 & 0.00 \\
\hline
\end{tabular}

and on this basis an equivalent inversion formula for Eq. (18), which is itself an approximation, becomes

$$
\theta_{0}=\frac{1}{2} B\left\{\frac{12}{B^{2}+12}+\frac{B^{2}\left[\pi-\frac{12 B}{B^{2}+12}\right]}{B^{3}+\beta}\right\} .
$$

This equation is valid for $\theta_{0}$ when $B \simeq 0$ and $B \rightarrow \infty$, independently of $\beta$. In order for Eqs. (18) and (20) to be consistent, $\beta \simeq 110$, as shown in Table II. It seems reasonable that a more refined approximation, such as least squares, could be constructed by including additional parameters, but since a more important quantity of interest is $y_{0} / l$, Eq. (20) will not be pursued further. Its main advantage, however, is that $\theta_{0}$ can be estimated directly in terms of $B$.

The same procedure applies to the approximation for $y_{0} / l$ which does not depend on Eq. (20). For this case the substitution of Eq. (15a) into Eq. (17) shows that $y_{0} / l$ has a small quadratic factor which can be neglected. From Eq. (2) it is observed that the integral factor of $2 / \sqrt{ } B$, for $\theta_{0}=\pi / 2$ has the value $1-\sqrt{ } 2 / 2$, so that for large values of $B$

$$
y_{0} / l \simeq 1-(0.586 / \sqrt{ } B) .
$$

Since $y_{0} / l \simeq B / 3$ for values of $B<1$ and Eq. (21) is valid for $B>9$, which can be verified by comparison with the exact solution, it can be concluded that

$$
\frac{y_{0}}{l}=\frac{B}{3}\left[\exp -\left(B^{2}+0.65 B\right)+\frac{3 B}{B^{2}+1}\left(1-\frac{0.586}{(B+0.344)^{1 / 2}}\right)\right],
$$

where suitable parameters have been introduced in order to match the exact values of $y_{0} / l$ for $B=3$ and $B=9$.

Table III shows a comparison of numerical results, obtained from Eqs. (18), (19), (20) and (22), with the exact values of [1]. The small differences in the values of $\theta_{0}$ from Eqs. (18) and (20) were neglected in the computation of $x_{0}$.

3. Conclusions. By accounting for second-order terms and the asymptotic properties of the elliptic integral solution, it can be concluded that:

1) the linear theory is entirely adequate for $0 \leq B \leq 1$.

2) for small values of $B(\leq 1.0), \theta_{0} \simeq \frac{1}{2} B\left(1-\frac{1}{12} B^{2}\right)$.

2 This procedure was suggested by Dr. H. Bueckner.

8 The constant 0.344 makes the term in parentheses vanish when $B=0$. 
TABLE III

\begin{tabular}{|c|c|c|c|c|c|c|}
\hline B & $\begin{array}{c}\theta_{0}^{9} \\
\text { Eq. (18) }\end{array}$ & $\begin{array}{c}\theta_{0}^{\prime} \\
\text { Eq. }(20)\end{array}$ & $\begin{array}{c}x_{0} / l \\
\text { Eq. (19) }\end{array}$ & $\begin{array}{c}y_{0} / l \\
\text { (Eq. 22) }\end{array}$ & $\begin{array}{c}x_{0} / l \\
\text { Ref. [1] }\end{array}$ & $\begin{array}{c}y_{0} / l \\
\text { Ref. [1] }\end{array}$ \\
\hline 0 & 0 & 0 & 1.00 & 0 & 1.00 & 0 \\
\hline 0.25 & 7.0 & 7.0 & 0.99 & 0.081 & 0.99 & 0.08 \\
\hline 0.5 & 14.1 & 14.1 & 0.99 & 0.166 & 0.98 & 0.16 \\
\hline 1.0 & 27.0 & 27.0 & 0.97 & 0.310 & 0.95 & 0.31 \\
\hline 2.0 & 46.5 & 46.2 & 0.86 & 0.496 & 0.84 & 0.49 \\
\hline 3.0 & 58.5 & 57.0 & 0.76 & 0.610 & 0.74 & 0.61 \\
\hline 6.0 & 74.0 & 74.2 & 0.56 & 0.746 & 0.56 & 0.74 \\
\hline 9.0 & 79.7 & 82.0 & 0.47 & 0.80 & 0.47 & 0.80 \\
\hline$\infty$ & 90.0 & 90.0 & 0.00 & 1.000 & 0.00 & 1.00 \\
\hline
\end{tabular}

3) the approximate formulas (18), (19), (20) and (22) are valid within $2 \%$ for $1 \leq$ $B \leq \infty$. Some improvement in these approximations might be achieved by including additional parameters.

4) it is not necessary to invert transcendental equations in order to obtain $x_{0}, y_{0}$, and $\theta_{0}$ since they are all expressed in terms of $B$.

5) all of the approximations, which have a rational basis, are simple, compact, and well adapted to slide-rule computation; no tables are required.

6) The success of the asymptotic method for this problem depends on matching both first and second derivatives at the origin as well as the behavior for $B=\infty$.

Appendix. ${ }^{4}$ asymptotic behavior of $\theta_{0}$. Although Eq. (20) approximates $\theta_{0}$ very well from $0 \leq \theta<\pi / 2$, the values of $\theta_{0}$ very near to $\pi / 2$ are not precisely those which would be obtained by the rigorous analysis which follows.

Let $K$ be the complete elliptic integral of the first kind with modulus $k$, and $F$ the hypergeometric function; then [2]

$$
K=\frac{1}{2} \pi F\left(\frac{1}{2}, \frac{1}{2}, 1 ; k^{2}\right), \quad F(a, b, c ; x)=\frac{\Gamma(a+b)}{\Gamma(a) \Gamma(b)} \log \frac{1}{1-x}
$$

for $a+b-c=0$. Now

$$
\frac{1}{1-k^{2}}=\frac{2}{1-\sin \theta_{0}}=\frac{2}{1-\cos \left(\frac{\pi}{2}-\theta_{0}\right)}=\frac{16}{\pi^{2}\left[1-\frac{2 \theta_{0}}{\pi}\right]^{2}} .
$$

For $\theta_{0} \simeq \pi / 2$

$$
\sqrt{ } B=\frac{1}{2} \log \frac{16 / \pi^{2}}{1-\left(\frac{2 \theta_{0}}{\pi}\right)^{2}} ; \quad \Gamma(1)=1 ; \quad \Gamma\left(\frac{1}{2}\right)=\sqrt{ } \pi ;
$$

hence

$$
\theta_{0} \simeq \frac{\pi}{2}-2 \exp (-\sqrt{ } B) \text { as } B \rightarrow \infty .
$$

4 This procedure was suggested by Dr. H. Bueckner. 
Eq. (20) is an algebraic approximation for $\theta_{0}$ which does not have the same asymptotic form as Eq. (A), but this is of no practical consequence since $x_{0}$ and $\theta_{0}$ are reasonably well approximated for $0 \leq B \leq 9$ by Eqs. (19) and (20), and for $B>9$

$$
x_{0} / l \simeq(2 / B)^{1 / 2} \text {. }
$$

\section{References}

[1] K. E. Bisshipp and D. C. Drucker, Large deflection of a cantilever beam, Quart. Appl. Math. 3, 272-276 (1945)

[2] E. T. Whittaker and Cr. N. Watson, A course in modern analysis, Cambridge University Press, Cambridge, 1927, pp. 297, 499 